\title{
Brain Stroke Segmentation using Fuzzy C-Means Clustering
}

\author{
S. Keerthana \\ Research Scholar \\ PSGR Krishnammal \\ College for Women \\ Coimbatore
}

\author{
K. Sathiyakumari \\ Assistant Professor \\ PSGR Krishnammal \\ College for Women \\ Coimbatore
}

\begin{abstract}
Image processing technique plays an important role in medical science for envisage various phenomenal structure of human body. Even though it helps more, sometimes it's very difficult to detect abnormal structures of human body by using simple images. Magnetic Resonance Imaging (MRI) is the one of the most significant technique to analyze human body and helpful for distinguishing and expounding the neural architecture of human brain effectively. This proposed strategy focus on detection and extraction of brain stroke from different patient's MRI images. In this work some preprocessing techniques like noise removal, filtering and segmentation is used for extract brain stroke partition accurately. The segmentation of brain stroke is implemented by using Fuzzy C-Means (FCM) clustering with two different levels of extraction. Edge detection is used for finding segmented portion of brain stroke edges accurately. Finally the stroke size is calculated for help doctors to make effective decisions about brain stroke. The experimental result proven that the proposed method is successful in detecting and extraction brain stroke efficiently with less time.
\end{abstract}

\section{Keywords}

FCM, MRI, CT, PET, hemorrhage, ischemic, embolic, WMF and etc.

\section{INTRODUCTION}

The techniques and processes of medical imaging are used to generate several human body parts for clinical purpose. In medical field, the quality of these images is plays an important role and the successful diagnosis of diseases mostly depends on the accuracy of segmentation algorithm [1],[2]. Most of the medical imaging related studies and diseases diagnosis are conducted by using Positron Emission Tomography (PET), Computed tomography (CT) and Magnetic Resonance Imaging (MRI) scan images [3]. MRI provides detailed information about diseases with multidimensional nature of different sequential pulses for giving accurate diagnosis and identifies many pathologic disease conditions.

Stroke occurs when blood supply to brain is interrupted or blocked. Stroke is also called brain attack. There are two main types of stroke: one is common form of stroke caused by blood clot/block in blood vessels and cause death of brain cells due to lack of oxygen. This type of stroke is called ischaemic stroke may occur in two ways: first one is thrombotic stroke occurs because of numerous reasons like cholesterol and fat [4]. It happens when the blood vessel becomes thin and the clot is stick on the wall of the artery [5], [6].
Second one is embolic strokes happen when the blood supply to the brain is stopped because of clot. Another one is deadly stroke which causes damage to human body due to bursting/bleeding into the brain. These types of stroke is called hemorrhage stroke. There are many types of hemorrhage stroke like cerebral, subarachnoid, epidural, intraparenchymal and subdural hemorrhage stroke [7]. It differs in many characteristic like stroke shape, location within skull and size of the stroke region.

MRI scan shows the great details because it is useful for imaging soft tissue like brain and bone. It shows abnormalities of brain which are too small or the abnormal region of brain that cannot be seen clearly by CT scan imaging. There are many methods and programs used to segment medical image to assist doctors to investigative the diseases. The most effective method is Image segmentation by using Matlab which helps doctors to analyze disease or unmoral tissue. The image segmentation techniques are useful in identification and isolation of an image into regions. Segmentation of brain using MRI scan image is complicated task. The following figure (Fig1) shows the normal and stroke infected MRI brain images.

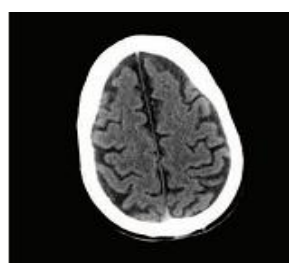

a) Normal Brain

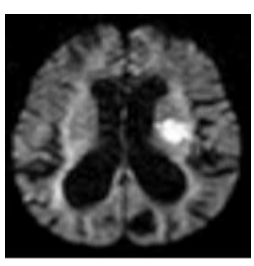

b) Ischemic Stroke

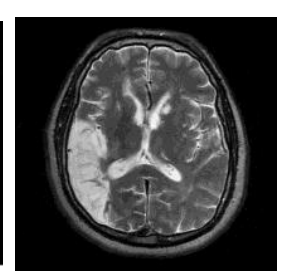

c) Hemorrhage Stroke
Fig1. MRI images normal and stroke images

Various types of segmentation methods are used in Matlab software like morphological and thresholding [8] which are work for find some type of brain stroke. One of the most common pixel-based segmentation methods is thresholding. In this method abnormal parts are segmented based on the intensity values of pixels. Morphological operations like dilation and erosion are nonlinear methods, it does with processing shapes. Process from erosion to dilation is remove background and from dilation to erosion is fill small holes and smoothens the object [9], [10]. In this work Fuzzy C-Means (FCM) clustering algorithm is used to segment the brain stroke region from MRI brain image.

The remaining paper is organized as follows; Section II portrays the proposed work of brain stroke segmentation. Section III gives the methodology used to extract the stroke 
region. Section IV demonstrates the result of segmentation process for splitting stroke region from MRI image using proposed algorithm. Finally section V describes the conclusion and future work of brain stroke detection.

\section{PROPOSED WORK}

The proposed work includes four different phases. The first and important phase is data collection; the MRI images of different patient's data are collected from reputed hospital lab in Coimbatore area for study purpose. The second and main phase for image processing is pre-processing the input image with various techniques like filtering and enhancement. Third phase is about segmentation of brain stroke region from original image using Fuzzy C-Means (FCM) algorithm. Final phase is calculating stroke size for help doctors to know about the stroke impacts clearly and display result image. The following figure (Fig2) illustrates the proposed framework for segmentation of brain stroke.

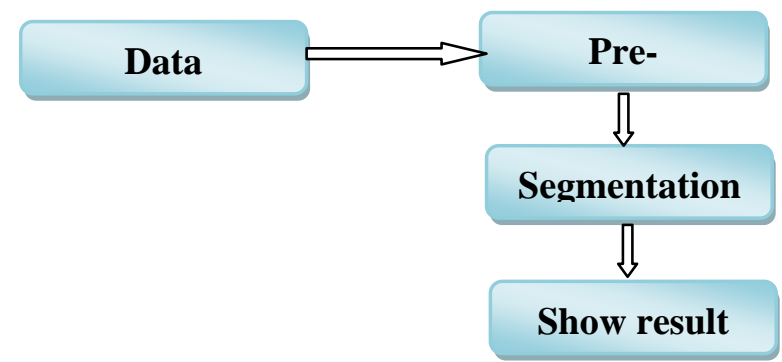

Fig1. Proposed framework for brain stroke segmentation

\subsection{Data Collection}

Data collection is the most important part in every work. In this research the data are collected from reputed hospital in Coimbatore. For each patient the MRI scan images are captured with particular time period. Every patient has 34 images in specific time interval with various pixel levels. The image has high resolution like $1105 \times 649$ pixels, which means each size is around $81 \mathrm{~KB}$.

\subsection{Pre-Processing}

The images are resized with same dimensions of width 256 pixels and height 256 pixels, vertical and horizontal resolutions of 96dpi and the bit depth is 24 . This process is done by using imresize() matlab function. The first process of preprocessing is removal of film artifacts from MRI images using roifill() matlab function and the second step is to extract skull portion of the brain image by using bwareaopen(),imfill() and imerode() matlab functions. To remove noise salt and pepper noise from MRI image Weighted Median Filtering (WMF) is used for segmentation easier and accurate.

\section{METHODOLOGY}

The methodology used to segment brain stroke region is Fuzzy C-Means (FCM) clustering algorithm, explained below:

\subsection{Fuzzy C-Means Clustering}

Fuzzy c-means has been a very significant tool for image processing in clustering image objects. FCM clustering is an unsupervised technique that has been effectively applied to clustering, feature analysis, and classifier designs in the fields of geology, target recognition, astronomy, medical imaging and image segmentation. This algorithm observe to analyze based on the distance among the different input data points. The clusters are created according to the distance between data points and cluster centers are created for each cluster [11],[12]. The Fuzzy C-Means Clustering algorithm is formulated as follows;

1. Let us consider an MRI brain input image composed of set of $\mathrm{N}$ points.

2. Let us suppose that this volume has to be segmented into $K$ classes, i.e $K \geq 2$ in fuzzy fashion.

3. It means that a point I does not necessarily belong to one of the $\mathrm{K}$ classes but can partially belong to quite a lot of ones.

4. For each point in $i \in N$, let $\left(u_{i c}\right)_{c=1}^{k}=\left(u_{i 1}, u_{i 2}, \ldots ., u_{i K}\right)$ the memberships of the point $\mathrm{I}$ with respect to these $\mathrm{k}$ classes, such that

$$
\sum_{c=1}^{K} u_{i c}=1 \text { and } u_{i c} \in[0,1]
$$

5. For each class $\mathrm{c}$ let $\mathrm{vc}$ be the centroid of this class. In FCM approach, the segmentation process of the image can be defined as the minimization of the energy function

$$
J_{F C M}=\sum_{c=1}^{K} \sum_{i=1}^{N} u_{i c}^{m}\left\|y_{i}-v_{c}\right\|^{2}
$$

6. The parameter $\mathrm{m}$ is a weighting exponent on each fuzzy membership and establishes the amount of ambiguity of the resulting classification. The equation (1) can be easily minimized using the lagrange multiplier $(\lambda)$, so the constrained optimization becomes as follows;

$$
F_{F C M}=\sum_{c=1}^{K} \sum_{i=1}^{N} u_{i c}^{2}\left\|y_{i}-v_{c}\right\|^{2}+\lambda\left(1-\sum_{c=1}^{K} u_{i c}\right)
$$

7. A solution can be obtained by alternatively calculating the membership ratios of uic and the centroids vc until convergence as follows:

$$
\begin{gathered}
v_{c}=\frac{\sum_{i=1}^{N} u_{i c}^{2} y_{i}}{\sum_{i=1}^{N} u_{i c}^{2}} \\
u_{i c}=\frac{1 / d_{i c}}{\sum_{j=1}^{K} 1 / d_{i j}}
\end{gathered}
$$

Where

$$
d_{i c}=\left\|y_{i}-v_{c}\right\|
$$

8. The memberships are often initialized with random values between 0 and 1 , such that the constraint of the membership is satisfied. The FCM function is minimized when high membership values are assigned to point whose intensities are close to the centroid of its fussy class, and low membership values are assigned when a point's intensity is far from the centroid.

\section{RESULTS}

The brain stroke detection is implemented in Matlab 2012a software. Various algorithms are used to segment and extract 
the stroke region from MRI image. First the images are resized with $256 \times 256$ pixels.

\subsection{Film Artifacts Removal}

MRI brain images have film artifacts or labels like patient's name, age, date, time, comment and etc. The labels are removed by using roifill() function in Matlab. The subsequent figure (fig3) shows the original image and film artifacts removed image.

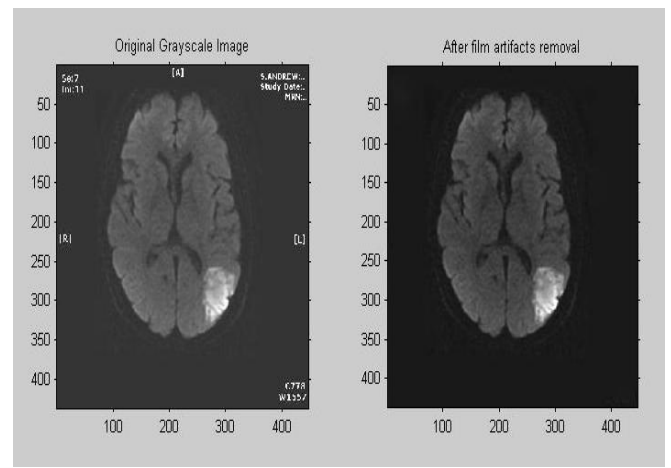

Fig3. Film Artifacts Removal

\subsection{Skull Extraction}

In this skull extraction phase unwanted partitions from MRI image is removed using bwareaopen(), imfill() and imerode() matlab functions. The following (Fig4) demonstrate the skull extracted image.

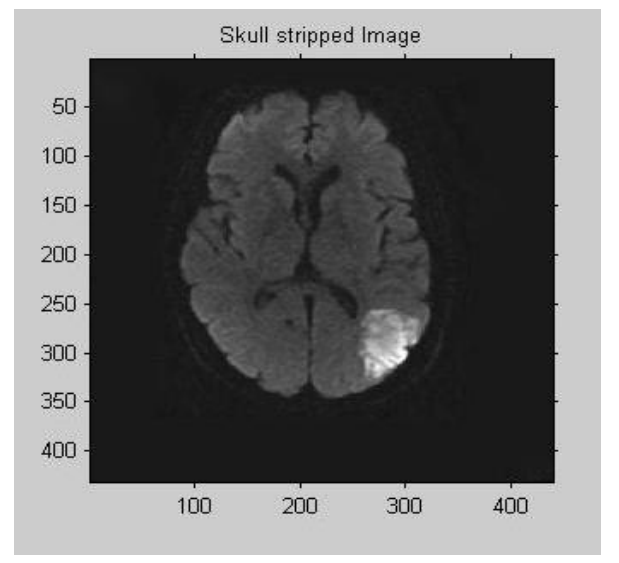

Fig4. Skull extracted image

\subsection{Weighted median filtering}

In the film artifacts removal the image salt and pepper noise because the film artifacts are removed based on the intensity values of the pixels. The weighted median filtering is used to remove salt and pepper noise. The following figure (Fig5) illustrates the weighted median filtering.

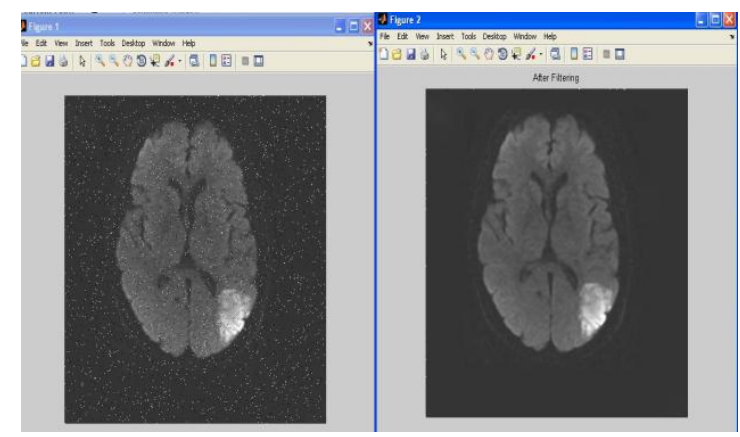

\section{Fig5.Weighted Median Filtering (WMF result image}

\subsection{Fuzzy C-Means Segmentation}

In this phase the brain stroke region is segmented in two levels and before applying fuzzy logic Otsu level thresholding is performed. FCM use using a 3-class fuzzy c-means clustering. It often works better than Otsu's method which outputs larger or smaller threshold on fluorescence images. The value of sw is 0 or 1 , a switch of cut-off position. If $\mathrm{sw}=0$, cut between the small and middle class and the $\mathrm{sw}=1$, cut between the middle and large class. The following figure (Fig6) shows the segmentation of brain stroke region in different levels.

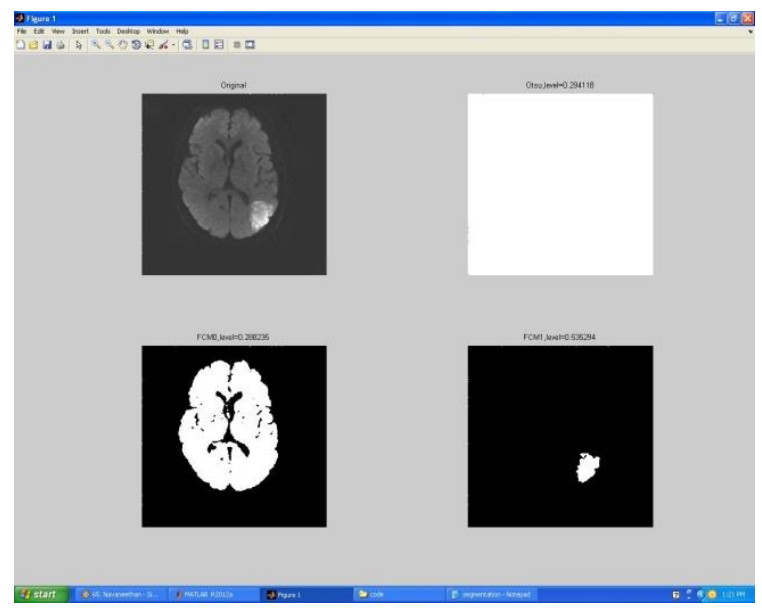

Fig6. Segmentation using Fuzzy C-Means Clustering

\subsection{Stroke Detection and Size calculation}

The segmented portion of brain stroke region is clearly extracted by using edge detection algorithm. The prewitt() function is used to detect the edge and the detected edges are colored to differentiate stroke region effectively. Finally the stroke size is calculated for help doctors to make decision accurately. The following figure (Fig7) gives the result of edge detected stroke image and the table (Table1 in Appendix) illustrates the stroke size for various images of original and stroke segmented images.

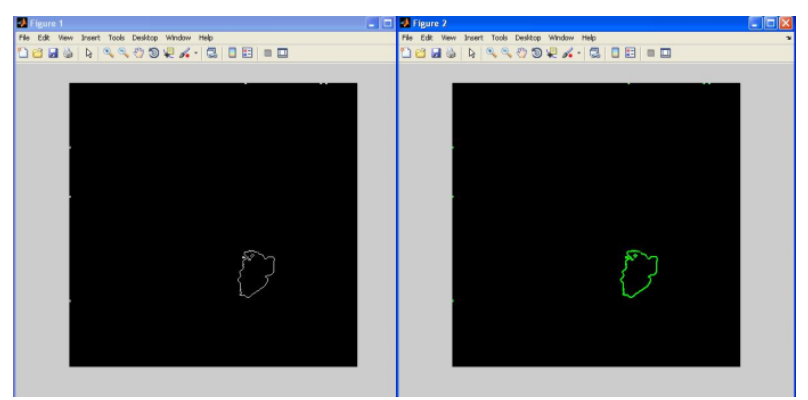

Fig7.Edge detection of brain stroke image

\section{CONCLUSION}

In this research work the procedure for the segmentation of MRI brain image to detect brain stroke. The brain stroke segmentation process is complicated because it includes various type of process like filtering, enhancement and morphological operations. The Fuzzy C-Means clustering technique is used to segment out the stroke region from input MRI image in three different classes of clustering with different levels of thresholding. The experimental result shows that the proposed segmentation work was able to detect different types of brain stroke in different type of position 
accurately. Finally the stroke size is calculated based on pixel intensity values to make clearly and effective decisions. In future work, different pre-processing techniques and segmentation techniques are used for detecting brain stroke.

\section{REFERENCES}

[1] M. M. K y a w, "Computer-Aided Detection system for Hemorrhage contained region ", International Journal of Computational Science and Information Technology, Vol. 1, No. 1, PP: 11 -16, Feb 2013

[2] B. Chanda, D. D. Majumder, Digital Image Processing and Analysis, PHI Learning Private Limited, New Delhi, Isbn 978 -81-203 -4325-2, 2nd Edition, 2011.

[3] E. D. Seletchi, O. G. Duliu, "Image Processing and Data Analysis in Computed Tomography", Rom. Journ. Phys., Bucharest, Vol. 52, Nos. 5-7, PP: 667-675, 2006.

[4] H. P. Adams, B. H. Bendixen," Classification of Subtype of Acute Ischemic Stroke Definitions for Use in a Multicenter Clinical Trial", Journal of the American Heart Association, No. 24, PP: 35 -41, 1993.

[5] A. Devi, S. P. Rajagopalan, "Brain Stroke Classification Based on Multi- Layer Perceptron Using Watershed Segmentation and Gabor Filter", Journal of Theoretical and Applied Information Technology, Vo 1. 56 No. 2, PP: 410-416, Oct 2013.

[6] F. Zhu, "Brain Perfusion Imaging - Performance and Accuracy", Centre for Intelligent System and their Applications, School of Information, University of Edinburgh, 2012.
[7] R. Liu, C. L. Tan, T. Y. Leong, C. K. Lee,B. C. Pang, C. Lim, Q. Tian, S. Tang, andZ. Zhang. Hemorrhage slices detection in brainct images. In19th International Conference onPattern Recognition (ICPR 2008), pages 1 -4, dec. 2008.

[8] Soltanian-Zadeh, H., Windham, J. P., \& Robbins, L. (1997, April). Semisupervised segmentation of MRI stroke studies. In Medical Imaging 1997 (pp. 437448). International Society for Optics and Photonics.

[9] Dougherty, E. R., Lotufo, R. A., \& The International Society for Optical Engineering SPIE. (2003). Hands-on morphological image processing (Vol. 71). Bellingham: SPIE press.[5]

[10] Abubakar, F. M. (2013). Study of Image Segmentation using Thresholding Technique on a Noisy Image. International Journal.

[11] K.S. Ravichandran and 2B Ananthi (2009) , “ color skin segmentation using k-means cluster," International Journal of computational and applied mathematics ISSN 1819 Volume 4 Number 2, pp. 153-157

[12] A.Suman Tatiraju july-2008," image segmentation using k-means clustering, EM and Normalized cuts," Symposium of Discrete Algorithm.

\section{APPENDIX}

The below table includes original image, segmented region of stroke image, original area of input image, segmented area of stroke image and the average stroke size.

Table1. Stroke size for different strokes

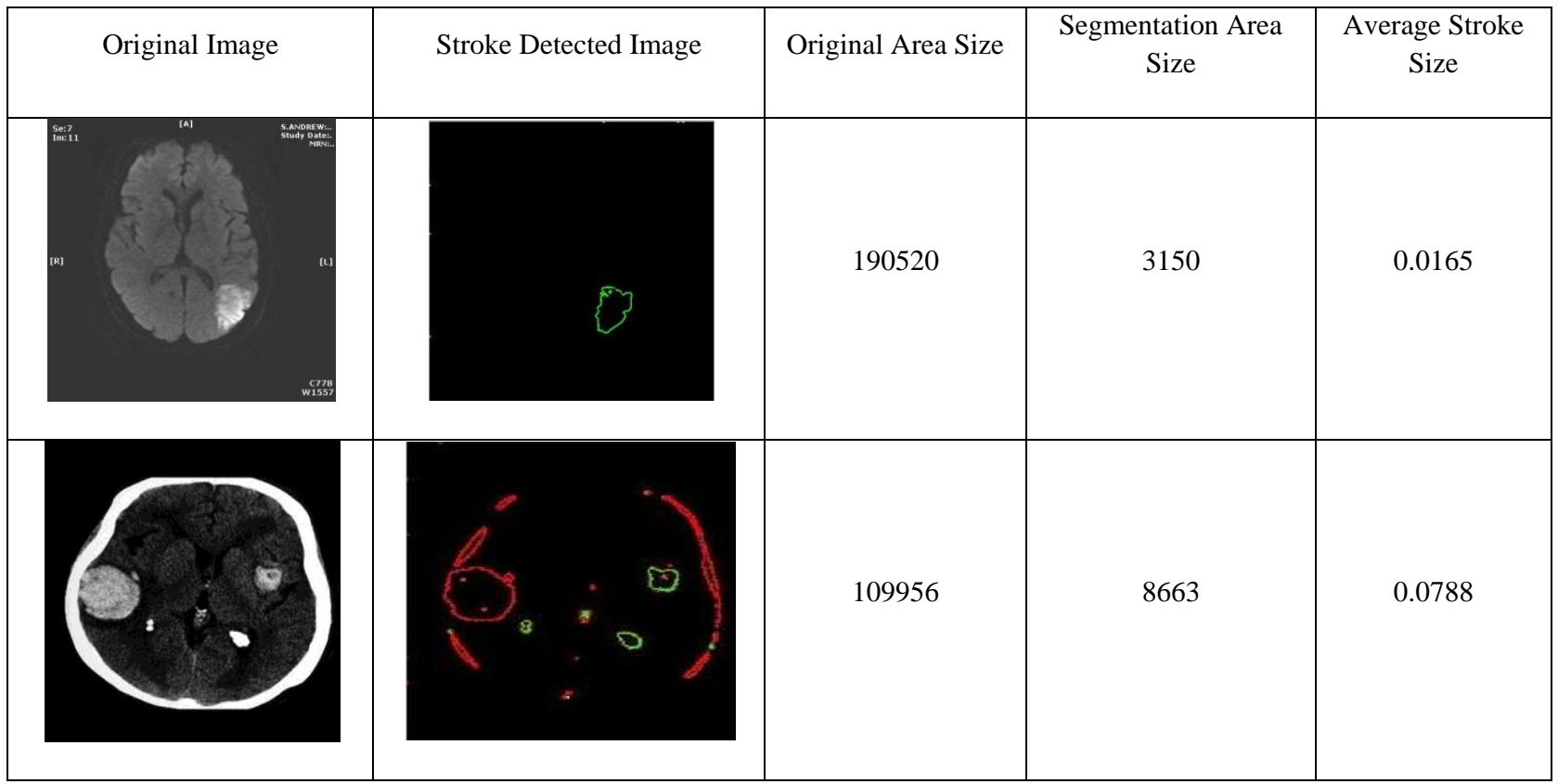


International Journal of Computer Applications (0975 - 8887)

Volume 154 - No.4, November 2016

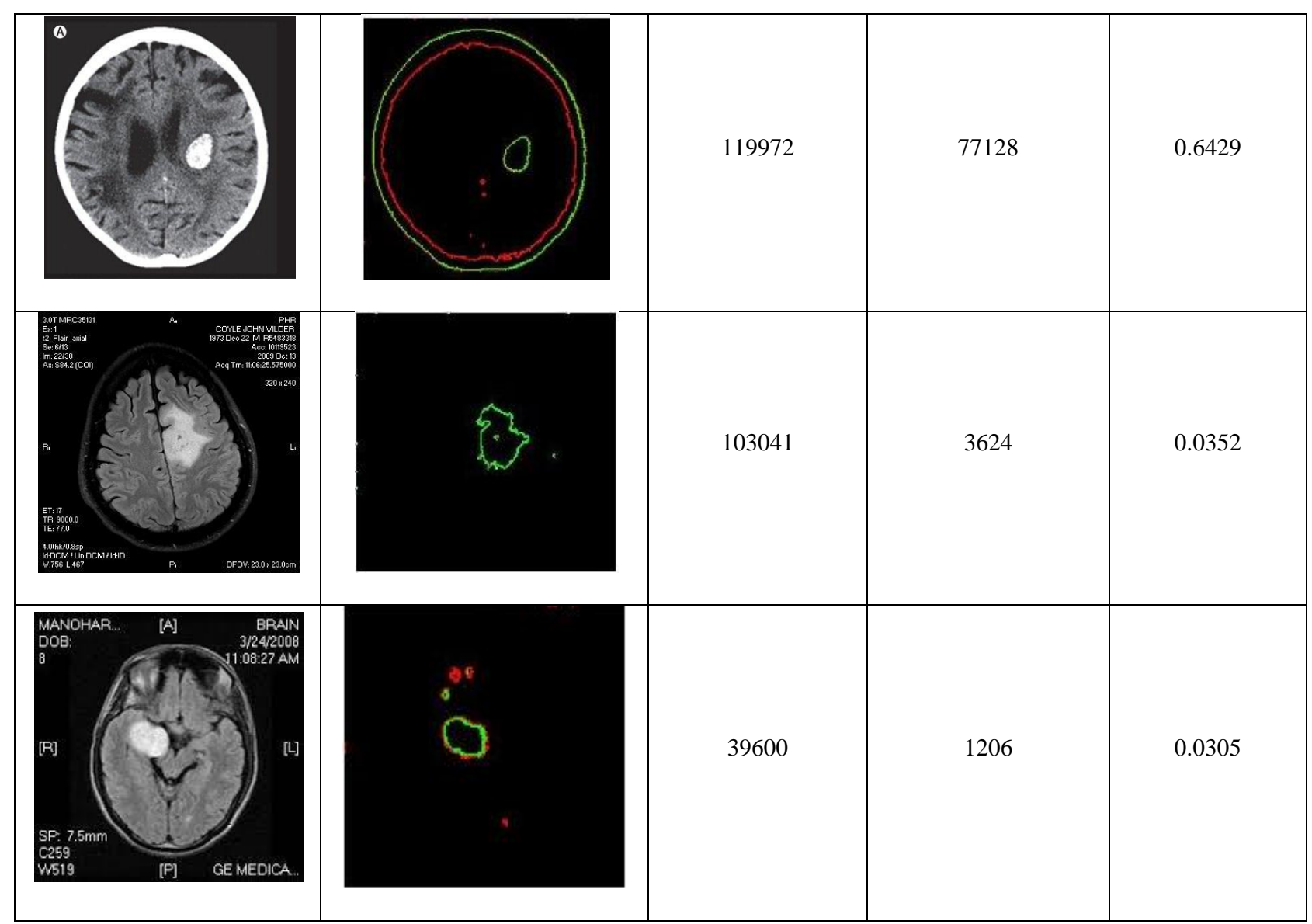

